\title{
Interferometric measurements of zonal winds on Titan
}

\author{
R. Moreno ${ }^{1}$, A. Marten ${ }^{1}$, and T. Hidayat ${ }^{2}$ \\ ${ }^{1}$ LESIA, Observatoire de Paris-Meudon, 5 place Jules Janssen, 92195 Meudon, France \\ e-mail: [raphael.moreno; andre.marten]@obspm.fr \\ 2 Department of Astronomy and Bosscha Observatory, ITB, Bandung, 40132, Indonesia \\ e-mail: taufiq@as.itb.ac.id
}

Received 5 October 2004 / Accepted 28 January 2005

\begin{abstract}
We report millimetre observations of Titan performed with the IRAM Plateau de Bure Interferometer at the rotational frequencies of $\mathrm{HC}_{3} \mathrm{~N}(25-24)$ and $\mathrm{CH}_{3} \mathrm{CN}(12-11)$. The most extended configuration of the array yielded disk-resolved emission spectra of Titan with an angular resolution of 0.6". Data were acquired in February-March 2003 and February 2004 near the greatest eastern and western elongations of Titan at high spectral resolution $\left(\lambda / \Delta \lambda=5.5 \times 10^{6}\right)$. Doppler lineshift measurements enable us to establish that the zonal wind flow is prograde in the upper atmosphere. Two different altitude regions are probed. Beam-integrated equatorial wind velocities of $160( \pm 60) \mathrm{m} / \mathrm{s}$ and $60( \pm 20) \mathrm{m} / \mathrm{s}$ are determined at respective altitudes of $300( \pm 150) \mathrm{km}$ (upper stratosphere) and $450( \pm 100) \mathrm{km}$ (lower mesosphere). The latter is the first direct determination of mesospheric wind speed on Titan.
\end{abstract}

Key words. planets and satellites: individual: Titan - techniques: interferometric - radio lines: solar system

\section{Introduction}

Most of the available information regarding Titan's planetaryscale meteorology has been obtained from observations by the Voyager 1 spacecraft in the late of 1980's. Titan's cyclostrophic winds of $100 \mathrm{~m} / \mathrm{s}$ have been inferred at $0.4-1.0$ mbar pressure levels (i.e., between 190- and 230-km altitude) from stratospheric temperature maps (Flasar et al. 1981; Flasar 1998). These measurements gave no conclusive evidence of the wind direction, however. Since the planet rotation is slow $(11.7 \mathrm{~m} / \mathrm{s})$, Titan's atmosphere is observed in superrotation, similar to that of Venus (Schubert 1983), the only other known case.

The 1989 occultation of the star 28 Sgr by Titan (Hubbard et al. 1993) showed an oblate atmosphere at a pressure level of 0.25 mbar (about $250-\mathrm{km}$ altitude). Stratospheric wind velocities of $100 \mathrm{~m} / \mathrm{s}$ were found around the equator, while higher values $(\sim 170 \mathrm{~m} / \mathrm{s})$ were estimated near the poles. These authors stated that their wind speed was accurate to an order of magnitude. In 2001, Titan's occultation by a binary star (Bouchez et al. 2003) yielded similar equatorial winds with velocities of $110 \pm 40 \mathrm{~m} / \mathrm{s}$ at $0.8 \mathrm{mbar}(200-\mathrm{km}$ altitude). High latitude jets of $230 \pm 20 \mathrm{~m} / \mathrm{s}$ and $160 \pm 40 \mathrm{~m} / \mathrm{s}$ are seen at $60^{\circ} \mathrm{N}$ and $40^{\circ} \mathrm{S}$ latitudes, respectively. These two indirect wind determinations did not give us any knowledge of the wind direction.

The first direct observations of winds on Titan were made by Kostiuk et al. (2001), who derived the wind direction from measurements of the Doppler line shifts of ethane lines at $12 \mu \mathrm{m}$, using infrared heterodyne spectroscopic techniques. A prograde atmospheric rotation was deduced, with equatorial wind speeds of $210 \pm 150 \mathrm{~m} / \mathrm{s}$ in the $0.1-7.0 \mathrm{mbar}$ pressure range ( $\sim 110-300 \mathrm{~km}$ altitude range). The velocity inferred from these measurements still had large error bars, however. Later on, Luz et al. (2003a) measured the Doppler line shifts of thousand of visible lines toward Titan's atmosphere, which also suggested that the wind is prograde.

Information on wind profiles of Titan's atmosphere yields important constraints on its atmospheric dynamics. Several general circulation models (GCMs) have been developed recently with numerical simulations. The first model (Hourdin et al. 1995) successfully simulated a superrotation of the middle atmosphere and consistently predicted prograde zonal winds on the order of 100-200 m/s in agreement with indirect determinations of winds by Flasar et al. (1981) and Hubbard et al. (1993). As shown by further models (Tokano et al. 1999; Rannou et al. 2004), the global atmospheric circulation of Titan also controls the distribution of gaseous constituents and aerosol haze and has strong seasonal variations. Dynamical transport in the thermosphere of Titan has been also investigated (Rishbeth et al. 2000; Müller-Wodarg et al. 2000). Wind speeds of $60 \mathrm{~m} / \mathrm{s}$ are predicted at altitudes of $\sim 1000 \mathrm{~km}$.

The present work deals with the first direct measurements of zonal winds on Titan from millimetre (mm) observations. Earlier studies done at these wavelengths (Marten et al. 2002) showed that narrow, isolated lines of the nitriles $\mathrm{HC}_{3} \mathrm{~N}$ and $\mathrm{CH}_{3} \mathrm{CN}$ are good candidates for probing the upper atmosphere of Titan. Using the same mm transitions, we now present a determination of the wind pattern in the upper 
stratosphere and lower mesosphere of Titan based on observations performed with the IRAM Plateau de Bure interferometer (PdBI). As we describe in Sect. 5, these observations allow us to probe two different altitude regions for the first time. A detailed spectral line study with the same data and devoted to the upper atmosphere composition will be presented in a forthcoming paper.

Sections 2 and 3 describe observations and data reduction, while Sects. 4 and 5 detail the Doppler line shift measurements and the wind field determination. Finally, our velocity results are discussed in Sect. 6, in the light of other wind measurements and compared with existing models of Titan's general circulation.

\section{Observations}

On February 23rd, March 10th 2003, and February 16th, 17th 2004, Titan was observed with the IRAM Interferometer, located in the French Alps at an altitude of $2500 \mathrm{~m}$. Observational parameters are presented in Table 1. The observing periods occurred in the southern summer for Titan.

The IRAM interferometer has six 15-m antennas, working at mm-wavelengths (Guilloteau et al. 1992). The most extended configuration was used with baseline lengths ranging from 32 to $400 \mathrm{~m}$.

Dual frequency receivers were used simultaneously at 3- and 1.3-mm, which gives access to the 82-116 and 210-245 GHz spectral ranges with system temperatures of 150 and $400 \mathrm{~K}$, respectively, thus characterizing the good observing conditions. Typical integration times were $\sim 7.5 \mathrm{~h}$ for each observing run.

Spectral maps were obtained in the $\mathrm{HC}_{3} \mathrm{~N}(J=25 \rightarrow 24)$ and $\mathrm{CH}_{3} \mathrm{CN}(J=12 \rightarrow 11)$ rotational lines at $1.3 \mathrm{~mm}$. Observations of the $\mathrm{HC}_{3} \mathrm{~N}(J=12 \rightarrow 11)$ and $\mathrm{HCN}(J=1 \rightarrow$ $0)$ transitions at $3-\mathrm{mm}$ wavelength were also performed. The $\mathrm{CO}(J=2 \rightarrow 1)$ broad-band feature at $230.5380 \mathrm{GHz}$ and the $\mathrm{HC}_{3} \mathrm{~N}(J=25 \rightarrow 24)$ narrow-band feature were recorded at the same time in the lower- and upper- sidebands of the $1.3-\mathrm{mm}$ receiver. Since all these lines were observed previously with the IRAM 30-m telescope (Hidayat et al. 1997, 1998; Marten et al. 2002), a precise evaluation of expected PdBI measurements could be made from our full-disk spectra.

The spectrometer (a correlator) had spectral resolutions of $40 \mathrm{kHz}$ for the $\mathrm{HC}_{3} \mathrm{~N}$ lines and 40,160 and $2500 \mathrm{kHz}$ for the $\mathrm{CH}_{3} \mathrm{CN}$ lines. The $\mathrm{HCN}$ and $\mathrm{CO}$ lines were recorded at 40 and $2500 \mathrm{kHz}$. The highest resolving power, $\lambda / \Delta \lambda=5.5 \times 10^{6}$, was obtained in the $\mathrm{HC}_{3} \mathrm{~N}(J=25 \rightarrow 24)$ and $\mathrm{CH}_{3} \mathrm{CN}(J=12 \rightarrow$ 11) measurements.

The elliptical Gaussian synthesized beam was $\sim 0.84 \times$ $0.45^{\prime \prime}$ at $1.3 \mathrm{~mm}$, with the long axis oriented roughly toward the north. This beam, smaller than Titan's mesospheric diameter ( 1 " at an altitude of $600 \mathrm{~km}$ ), was therefore sufficient to resolve Titan. At $3 \mathrm{~mm}$, the synthesized beam was $\sim 1.8 \times$ $1.1^{\prime \prime}$, which only yielded whole disk average measurements. The beam parameters are summarized in Table 2.

Since Titan is tidally phase-locked with Saturn, groundbased telescopes give observations of the same side of the moon at a given elongation. Our 2003 observations covered
Table 1. Observational parameters for Titan.

\begin{tabular}{|c|c|c|c|c|}
\hline Date & $\begin{array}{l}\text { Surface } \\
\text { angular } \\
\text { diameter } \\
\left({ }^{\prime \prime}\right)\end{array}$ & $\begin{array}{c}\text { Geocentric } \\
\text { distance } \\
(\mathrm{AU})\end{array}$ & $\begin{array}{l}\text { Sub-earth } \\
\text { latitude }\end{array}$ & $\begin{array}{l}\text { North } \\
\text { polar } \\
\text { angle } \\
\left(^{\circ}\right)\end{array}$ \\
\hline 2003-Feb.-23 & 0.817 & 8.68945 & -26.58 & 355.12 \\
\hline 2003-Mar.-10 & 0.795 & 8.93708 & -26.61 & 355.10 \\
\hline 2004-Feb.-16 & 0.848 & 8.37370 & -25.82 & 353.97 \\
\hline 2004-Feb.-17 & 0.846 & 8.38928 & -25.82 & 353.97 \\
\hline
\end{tabular}

Table 2. Frequency setup and synthesized beam.

\begin{tabular}{|c|c|c|c|c|}
\hline \multirow[b]{2}{*}{ Date } & \multirow{2}{*}{$\begin{array}{l}\text { Observed } \\
\text { transition }\end{array}$} & \multirow{2}{*}{$\begin{array}{c}\text { Line } \\
\text { frequency } \\
(\mathrm{GHz})\end{array}$} & \multicolumn{2}{|l|}{ Beam } \\
\hline & & & $\begin{array}{l}\text { Size } \\
\left({ }^{\prime \prime}\right)\end{array}$ & $\begin{array}{l}\text { PA } \\
\left({ }^{\circ}\right)\end{array}$ \\
\hline \multicolumn{5}{|l|}{2003} \\
\hline \multirow[t]{3}{*}{ Feb.-23 } & $\mathrm{HC}_{3} \mathrm{~N}(25 \rightarrow 24)$ & 227.418906 & $0.80 \times 0.47$ & 18 \\
\hline & $\mathrm{CO}(2 \rightarrow 1)^{a}$ & 230.538000 & $0.78 \times 0.45$ & 18 \\
\hline & $\mathrm{HC}_{3} \mathrm{~N}(12 \rightarrow 11)$ & 109.173634 & $1.77 \times 0.98$ & 16 \\
\hline \multirow[t]{2}{*}{ Mar.-10 } & $\mathrm{CH}_{3} \mathrm{CN}(12 \rightarrow 11)^{b}$ & 220.709080 & $0.89 \times 0.47$ & 14 \\
\hline & $\mathrm{HCN}(1 \rightarrow 0)^{c}$ & 88.631847 & $2.17 \times 1.20$ & 12 \\
\hline \multicolumn{5}{|l|}{2004} \\
\hline \multirow[t]{3}{*}{ Feb.-16 } & $\mathrm{HC}_{3} \mathrm{~N}(25 \rightarrow 24)$ & 227.418906 & $0.84 \times 0.44$ & 13 \\
\hline & $\mathrm{CO}(2 \rightarrow 1)^{a}$ & 230.538000 & $0.83 \times 0.43$ & 15 \\
\hline & $\mathrm{HC}_{3} \mathrm{~N}(12 \rightarrow 11)$ & 109.173634 & $1.62 \times 0.93$ & 14 \\
\hline \multirow[t]{2}{*}{ Feb.-17 } & $\mathrm{CH}_{3} \mathrm{CN}(12 \rightarrow 11)^{b}$ & 220.709080 & $0.82 \times 0.44$ & 15 \\
\hline & $\mathrm{HCN}(1 \rightarrow 0)^{c}$ & 88.631847 & $1.85 \times 1.21$ & 16 \\
\hline
\end{tabular}

Notes: PA denotes the position angle of the elliptical beam.

${ }^{a}$ Recorded in the Upper SideBand of the receiver at $230.5380 \mathrm{GHz}$.

${ }^{b}$ Five rotational transitions were observed at $160-\mathrm{kHz}$ resolution over a 80-MHz-wide bandwidth.

${ }^{c}$ The hyperfine lines (triplet) were observed at $40-\mathrm{kHz}$ resolution.

Titan's eastern elongation (leading hemisphere seen), and those done in 2004 covered Titan's western elongation (trailing hemisphere seen), in order to study possible changes between both hemispheres.

\section{Data reduction and calibration}

During all observing runs, amplitudes and phases of the visibilities were calibrated with observations of quasars every $20 \mathrm{~min}$. Pointing and focus were monitored regularly. Calibration was done as follows:

1. Sideband gain and bandpass calibration: measurements of the upper to lower sideband gain ratio, as well as the relative channel gain versus frequency, were done against the strong quasars $0420+014$ and 3C 273 within accuracies of $0.1 \%$ and $3 \%$, respectively.

2. Phase calibration: the absolute atmospheric and instrumental phase variations with time were evaluated by observing the quasars $0415+379,0528+134$ and $0748+126$. At $1.3 \mathrm{~mm}$, typical phase errors were $40^{\circ} \mathrm{rms}$. 
3. Amplitude calibration: the amplitude variation with time is measured by observing the same quasars. These variations were mainly due to atmospheric de-correlation, as well as to pointing and focus errors. The corresponding relative uncertainties are $\sim 10 \%$.

The followings additional steps were necessary in the data reduction because of the nature of the Titan observations.

a. The velocity follow-up procedure of the telescopes continuously corrects the Doppler velocity of Saturn relative to the Earth, but not that of Titan. We therefore introduced, a posteriori, accurate corrections of Titan's orbital velocity (relative to Saturn) taken from the JPL ephemeris (JPL Solar System Dynamics, website: http://ssd. jpl . nasa.gov). In practice, the velocity corrections were applied to the data by changing the corresponding velocity parameter for each individual scan and then resampling the data on the same velocity grid, before creating the uv-tables. This yielded spectra in an absolute velocity frame.

b. The image quality was improved by phase self-calibration techniques. It helps to correct the atmospheric phase error by referencing the phase to what is expected on the source. Such techniques are usually applied when a source like a planet (having a circular-disk shape) has a strong continuum flux that is easily modeled (Titan is $\sim 1.5 \mathrm{Jy}$ at $1.3-\mathrm{mm}$ ). In practice, phase self-calibration strongly reduces phase errors in the data. We found, for instance, that $1.3-\mathrm{mm}$ data measured with $40^{\circ}$ of phase rms (i.e., with an equivalent atmospheric seeing of $0.17^{\prime \prime}$ ) were improved to $5^{\circ}$ of phase rms (seeing of $0.02^{\prime \prime}$ ) after phase self-calibration.

c. The absolute flux is obtained by scaling the continuum sideband (free of lines) to our Titan's radiative transfer flux density model (from Hidayat et al. (1997) and Marten et al. (2002); See also Sect. 5).

d. The last step in data reduction was to create spectral maps from uv-tables. The channel maps were obtained from Fourier transforms of the visibilities for each spectral channel. These maps were then deconvolved from their associated dirty beams to produce clean maps with the standard "Clark" algorithm.

Final results are illustrated by the Titan continuum map in Fig. 1 and the spectral maps of the $\mathrm{HC}_{3} \mathrm{~N}(J=25 \rightarrow 24)$ and $\mathrm{CH}_{3} \mathrm{CN}(J=12 \rightarrow 11)$ features in Figs. 2 and 3. These plots show selected positions on Titan separated by $0.2^{\prime \prime}$, although interferometric measurements yield a full map of all points on Titan simultaneously. Averages of data, corrected for the varying geocentric distances of Titan, were done over the two observing periods of 2003 and 2004.

\section{Doppler lineshift measurements}

\subsection{Absolute frequency evaluation}

The best spatial resolution on Titan is obtained in the $\mathrm{HC}_{3} \mathrm{~N}(J=25 \rightarrow 24)$ and $\mathrm{CH}_{3} \mathrm{CN}(J=12 \rightarrow 11)$ lines. In

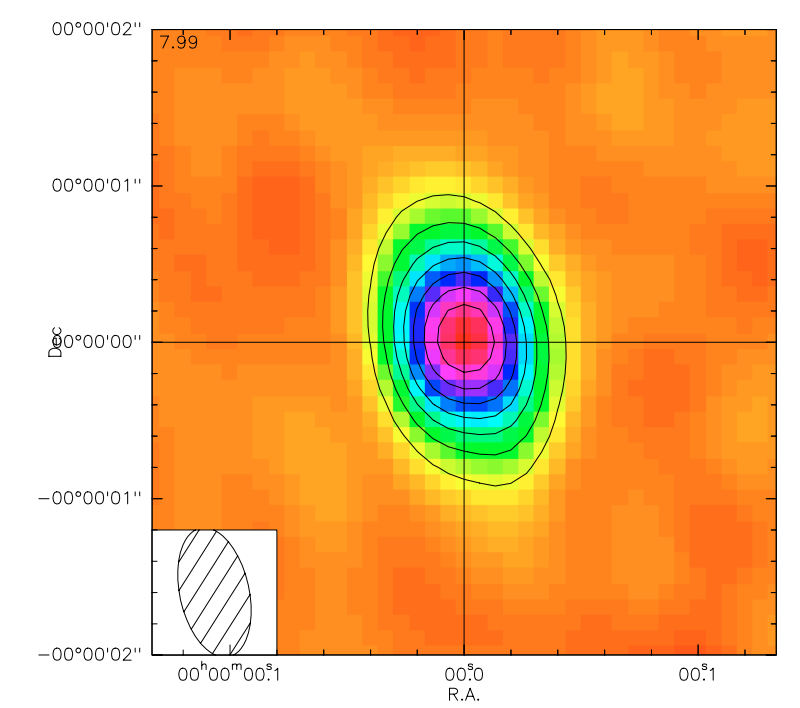

Fig. 1. Titan's continuum map observed at $227.4 \mathrm{GHz}$ in 2003 after phase self-calibration. Contour levels are plotted in intervals of $0.1 \mathrm{Jy}$. The dashed circle indicates the planetary surface.

them, determination of zonal wind is feasible from direct examination of frequency Doppler shifts observed at the highest spectral resolution.

In a first approach, we fit the line profiles of isolated lines with Gaussian profiles, which are sufficient because the line features are mainly Voigt-Doppler broadened. Along the meridian through the position $(0,0)$ we observed that the Doppler shifts were all different from zero, with figures well outside the expected range of frequency uncertainties in the line catalog (JPL catalog, Pickett et al. 1998). Frequency errors quoted in that catalog are about $1-4 \mathrm{kHz}$ for $\mathrm{HC}_{3} \mathrm{~N}$ lines; $150 \mathrm{kHz}$ mentioned for the $\mathrm{CH}_{3} \mathrm{CN}(J=12 \rightarrow 11)$ lines appear not to be really significant. Figures 4 and 5 show illustrations of the frequency shifts for four lines.

We checked that the velocity corrections (i.e., Titan's velocity correction and resampling done a posteriori, see Sect. 3) were accurate enough in all our data sets including data taken at $3 \mathrm{~mm}$. For completeness we also used the IRAM 30-m spectra recorded in 1999 at the same frequencies (Marten et al. 2002). Gaussian fits to the line profiles were computed to determine all the frequency offsets and associated errors, the JPL catalog frequencies serving as reference. We verified that another line catalog (Cologne Database CDMS, http://www.ph1.uni-koeln.de/vorhersagen/ Muller et al. 2001) gives identical line frequencies with similar uncertainties. Table 3 indicates the results of the line fitting.

Adopting the most recent molecular constants of $\mathrm{HC}_{3} \mathrm{~N}$ and $\mathrm{CH}_{3} \mathrm{CN}$ published by Mbosei et al. (2000) and Pearson \& Muller (1996), respectively, as well as the standard formalism for rotational transitions in the ground vibrational state (see for instance Brown et al. (1988), for $\mathrm{CH}_{3} \mathrm{CN}$ ), we computed the rotational frequencies of both compounds. We discovered that our computed frequencies were affected by smaller errors and differred slightly from those of the line catalogs. Table 3 shows our computations. Note that the $\operatorname{HCN}(J=1 \rightarrow 0)$ line 


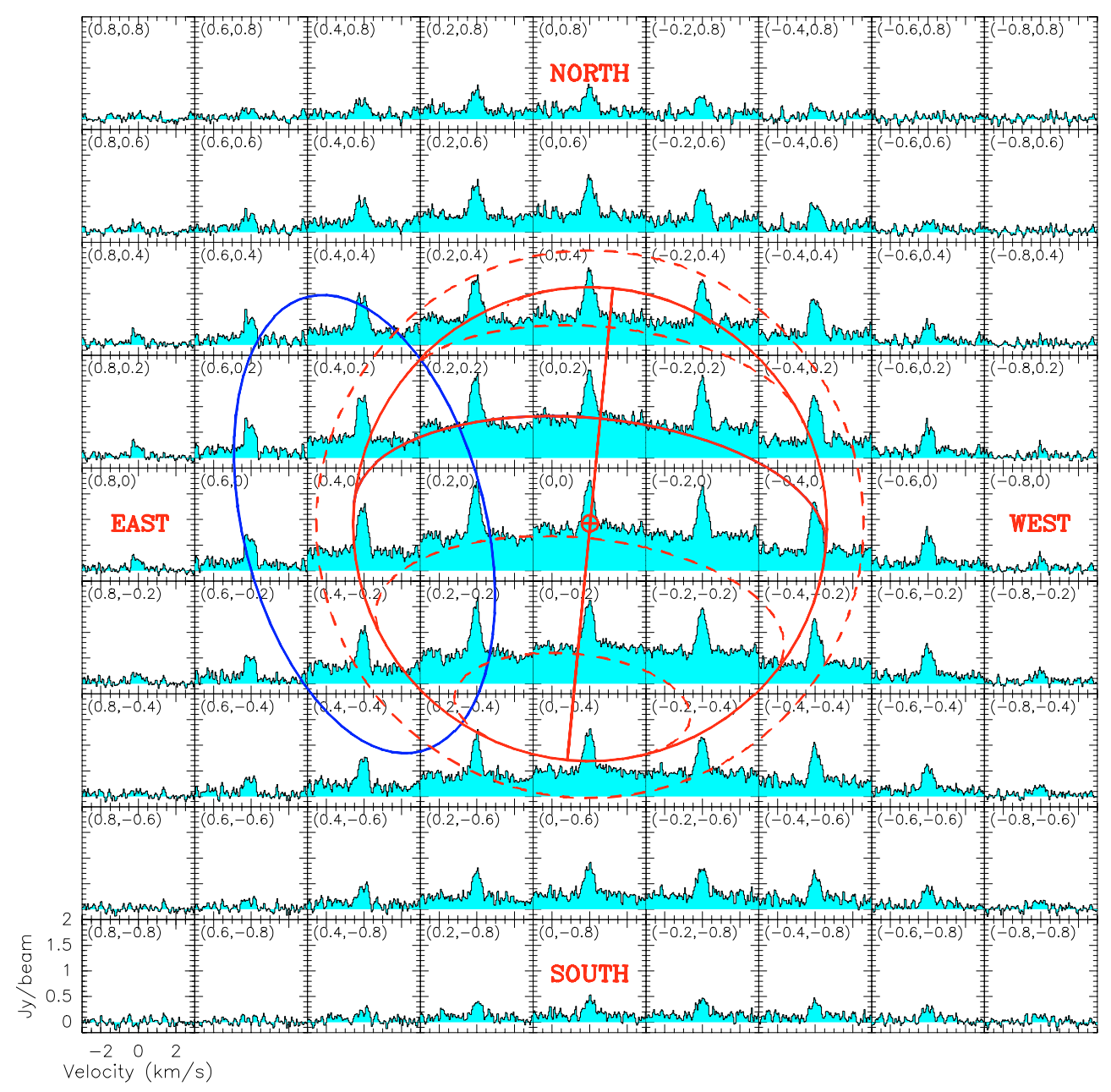

Fig. 2. A spectral map in the $\mathrm{HC}_{3} \mathrm{~N}(J=25 \rightarrow 24)$ transition. The usual units in radioastronomy measurements are used here. RA and Dec offsets (in ") are indicated for each position of the map, with Titan's centre at $(0,0)$. North is at the top. A weighted average was done over the 2003 and 2004 data to enhance the signal-to-noise ratio. The spectral resolution is $40 \mathrm{kHz}$. The solid circle corresponds to the planetary surface $(2575 \mathrm{~km})$. The dashed circle indicates the atmosphere at a mean altitude of $400 \mathrm{~km}$. The ellipse shows the synthesized beam at $1.3 \mathrm{~mm}$.

positions predicted by Nguyen-Van-Thanh \& Rossi (1993) are the same as those of the two line catalogs.

Most of the lineshifts are lower than the spectral resolution of $40 \mathrm{kHz}$ and all $1 \sigma$ associated uncertainties are smaller. This means that the absolute frequency of the observed lines are accurate at the level of the spectral resolution, (or channel spacing of the spectrometer). Relative frequency measurements are much more precise, on the order of these uncertainties, although errors affecting the resampling technique (about one half-channel bandwidth) are not negligible. As a first conclusion, we note that the line frequencies are correctly estimated at the lower frequencies, indicating that our velocity correction procedure is well suited to our measurements. Since the same procedure, with the same shift values, applies to the higher frequency lines, we can consider that the absolute higher frequencies are estimated precisely from the measured Doppler lineshift values given in Table 3 .

In a second approach, we computed synthetic spectra at the same spectral resolution of $40 \mathrm{kHz}$. The vertical distributions of nitrile abundances inferred from IRAM 30-m observations (Marten et al. 2002) served as initial values for radiative transfer computations considering a spherical geometry for Titan's atmosphere and an elliptical Gaussian synthesized beam. No Doppler velocity shift was assumed in the preliminary computations. The equatorial distributions proposed by Marten \& Moreno (2003) to explain the 2003 PdBI data were found to be similar, which meant that a direct comparison was possible between the observed and calculated features. Spectral differences and error estimates from a $\chi^{2}$ fitting were derived after changing the frequency reference of the synthetic spectra. Using this second method that in practice considers an iterative grid-search algorithm (Bevington \& Robinson 1992), frequency shifts were found on the same order as those shown in Table 3. Similar computations that include Doppler velocity shifts of the lines have been also considered below to derive the wind field on Titan's disk.

\subsection{Doppler velocity shift determination}

Doppler velocity shifts were computed for the whole map of Titan to determine the zonal wind field. Line fit uncertainty depends on both the signal-to-noise ratio in the line contrast and 


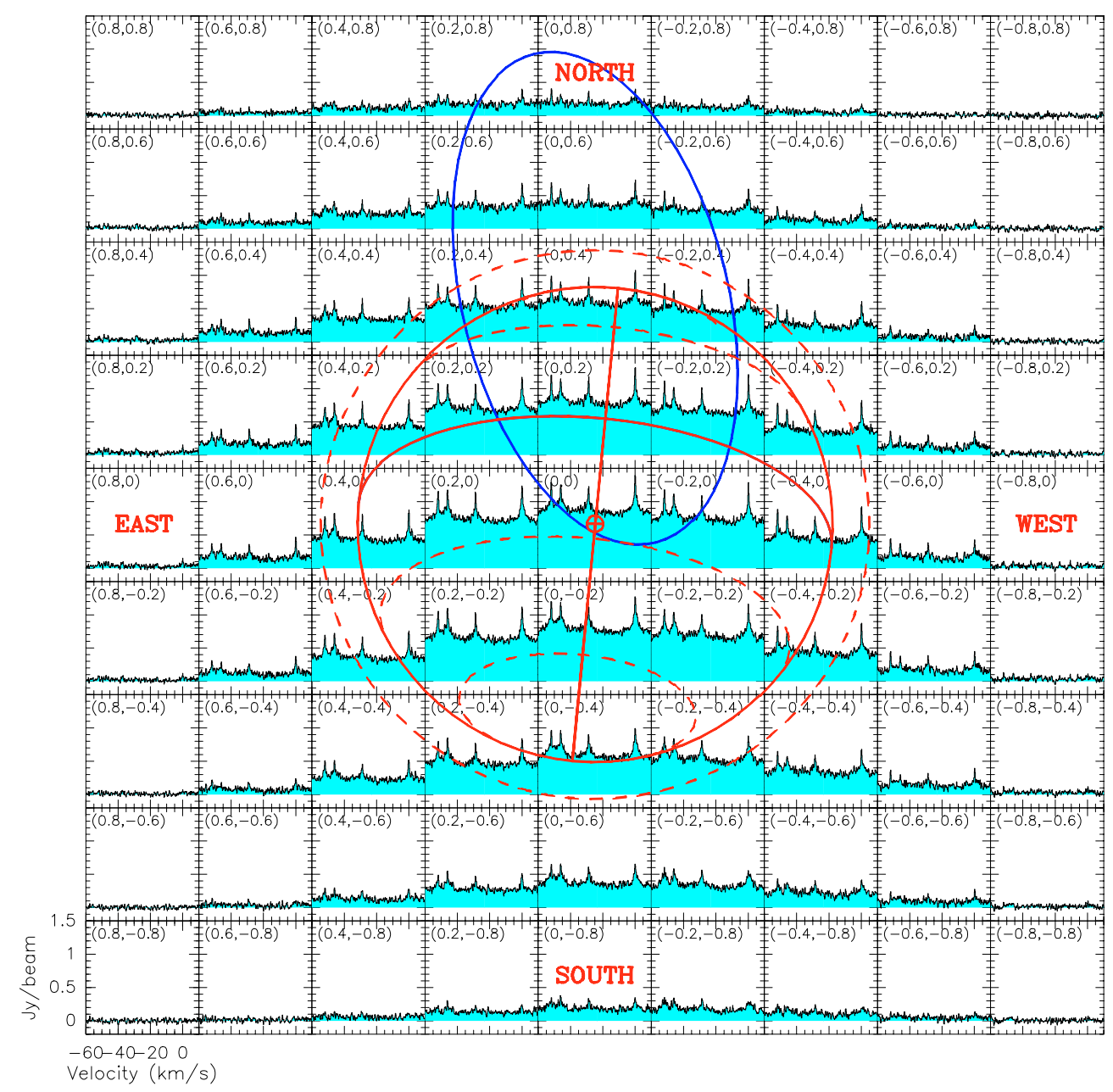

Fig. 3. Same as Fig. 2 but for $\mathrm{CH}_{3} \mathrm{CN}(J=12 \rightarrow 11)$ observations at a spectral resolution of $160 \mathrm{kHz}$.

on the channel bandwidth. The $\mathrm{HC}_{3} \mathrm{~N}(J=25 \rightarrow 24)$ feature at the highest correlator spectral resolution of $40 \mathrm{kHz}$ gave us the most accurate estimate. As the signal-to-noise ratio affecting one individual line of $\mathrm{CH}_{3} \mathrm{CN}(J=12 \rightarrow 11)$ taken at a spectral resolution of $40 \mathrm{kHz}$ was not high enough, we added the 4 strongest components $(K=0,1,2,3)$ recorded at a spectral resolution of $160 \mathrm{kHz}$ by shifting the corresponding central frequencies to $220.709080 \mathrm{GHz}$. This is possible because the four components probe the same altitude region (see Sect. 5 below).

Two different computation methods were used: Gaussian fitting and direct comparison of synthetic and observed line profiles. Consistent results have been obtained in both cases. Figure 6 illustrates the Gaussian fit results for both $\mathrm{HC}_{3} \mathrm{~N}$ and $\mathrm{CH}_{3} \mathrm{CN}$ lines. The values are not symmetric around the central meridian, as one could expect for a uniform zonal wind field, but it can be explained by the small line frequency errors indicated in Table 3. Doppler velocity shifts results are summarized in Table 4. From the $\mathrm{HC}_{3} \mathrm{~N}(J=25 \rightarrow 24)$ lineshift differences between the western and eastern equatorial limbs $(\Delta v)$, values are found equal to $+111 \pm 24$ and $+58 \pm 18 \mathrm{~m} / \mathrm{s}$ in 2003 and 2004, respectively. From the $\mathrm{CH}_{3} \mathrm{CN}(J=12 \rightarrow 11)$ data, higher values are determined, equal to $+191 \pm 42$ in 2003 and $+178 \pm 74 \mathrm{~m} / \mathrm{s}$ in 2004 . The positive difference we find clearly demonstrates a prograde zonal wind flow.
The measured $\Delta v$ through $\mathrm{HC}_{3} \mathrm{~N}$ seems to have varied between 2003 and 2004 but is also compatible at $1 \sigma$ with a mean value of $+85 \pm 20 \mathrm{~m} / \mathrm{s}$. An averaged Doppler velocity of $+185 \pm 40 \mathrm{~m} / \mathrm{s}$ is in agreement with both the 2003 and 2004 $\mathrm{CH}_{3} \mathrm{CN}$ measurements.

\section{Retrieval of the wind field}

Lineshifts were computed for each data point of Titan's maps. Since we observed that Gaussian fits were precise enough, simple calculations of Doppler velocity shifts were undertaken. Considering the potential line frequency uncertainties and assuming a constant zonal wind flow, mean lineshift values between the west and east limbs were removed in the Doppler velocity maps presented in Figs. 7a and 8 a.

On the other hand, the Doppler shift results that correspond to beam-averaged velocity values have to be converted into zonal wind velocities. For that purpose, we simulated the expected Doppler lineshifts on a rotating sphere, taking into account the observing geometry (related to the sub-earth point of $-26^{\circ}$, see Table 1) and the convolution of the emitted flux by the synthesized beam. We used our Titan radiative transfer code (Hidayat et al. 1997; Marten et al. 2002) and included a suitable geometrical interface code initially developed for 

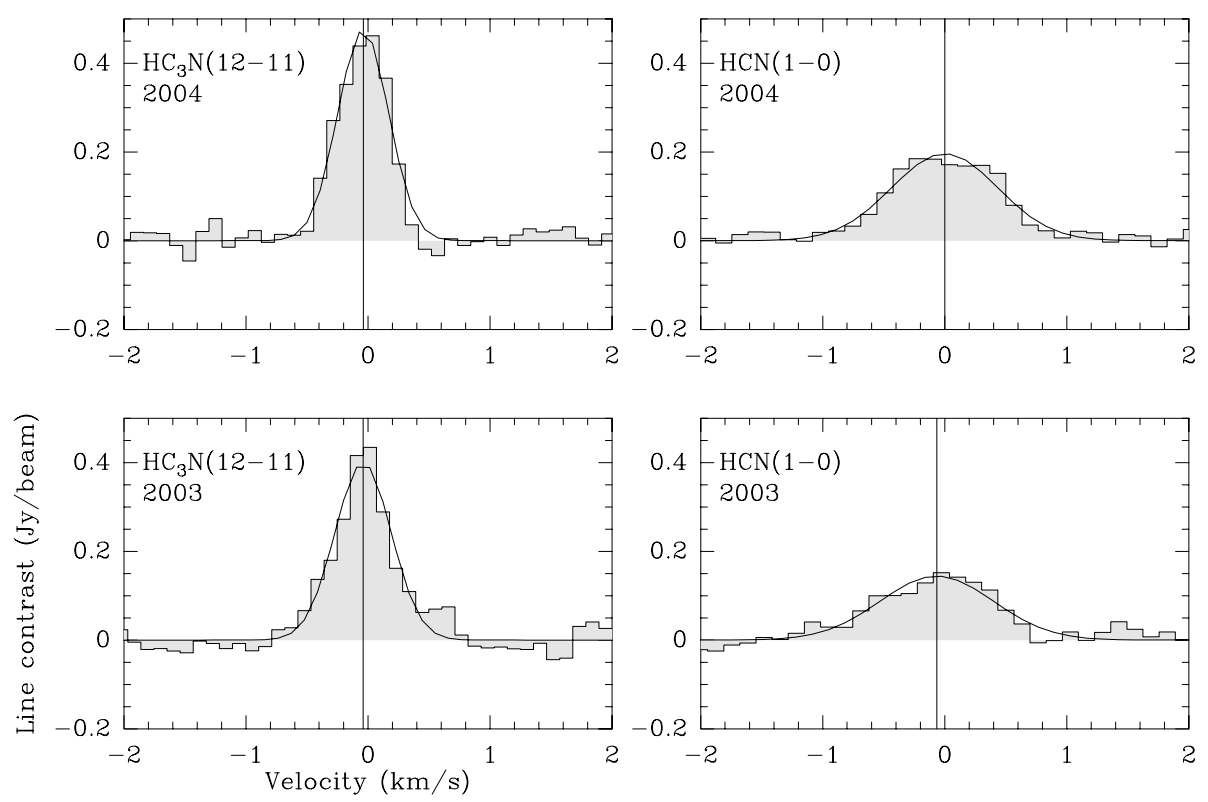

Fig. 4. $\mathrm{HC}_{3} \mathrm{~N}(J=12 \rightarrow 11)$ and $\mathrm{HCN}(J=1 \rightarrow 0)$ spectra observed at Titan's central position, with Gaussian fits superposed. Only one component of $\operatorname{HCN}(J=1 \rightarrow 0)$, centered at $88.631847 \mathrm{GHz}$, is shown. Intensity is expressed in line contrast because Titan's continuum emission has been subtracted (a zeroth-order baseline has been removed). The spectral resolution is $40 \mathrm{kHz}$. The vertical line marks the calculated velocity shift (Table 3).
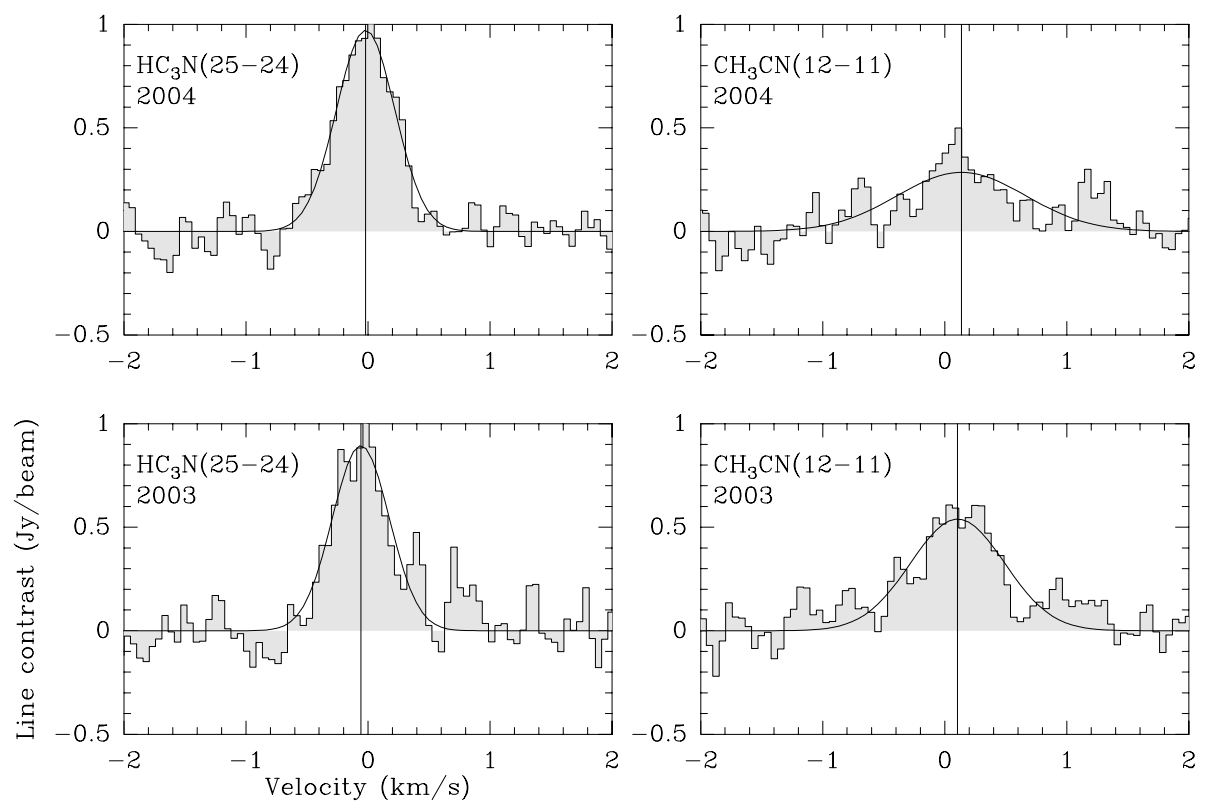

Fig. 5. Same as Fig. 4, but for $\mathrm{HC}_{3} \mathrm{~N}(25 \rightarrow 24)$ and $\mathrm{CH}_{3} \mathrm{CN}(12 \rightarrow 11, K=3)$.

Jupiter (Moreno et al. 2001). The results of two simulations corresponding to equatorial maximum velocities of +70 and $+170 \mathrm{~m} / \mathrm{s}$ are shown in Figs. $7 \mathrm{~b}$ and $8 \mathrm{~b}$. Note that the velocity contours vary slightly near the equatorial limbs where the maximum velocity Doppler shifts are determined.

After removing Titan's rotation velocity $(11.7 \mathrm{~m} / \mathrm{s})$, we finally obtain an average zonal wind of $+60 \pm 20 \mathrm{~m} / \mathrm{s}$ and $+160 \pm$ $60 \mathrm{~m} / \mathrm{s}$ from the $\mathrm{HC}_{3} \mathrm{~N}(J=25 \rightarrow 24)$ and $\mathrm{CH}_{3} \mathrm{CN}(J=12 \rightarrow$ 11) lines, respectively.
In order to determine the atmospheric levels probed by these wind measurements, contribution functions were computed for both lines with our best atmospheric model (Marten $\&$ Moreno 2003). Normalized contribution functions were computed first (Fig. 9a). Because the 4 strongest components $(K=0,1,2,3)$ of $\mathrm{CH}_{3} \mathrm{CN}(J=12 \rightarrow 11)$ probe almost the same altitude levels, they can therefore be coadded. Weighted contribution functions (Fig. 9b) were then computed using the line derivative for each frequency as a weight, averaged over 
Table 3. Doppler frequency lineshift measurements at Titan's centre.

\begin{tabular}{|c|c|c|c|c|}
\hline \multirow[t]{2}{*}{ Date } & \multirow[t]{2}{*}{ Transition } & \multicolumn{3}{|c|}{$\begin{array}{l}\text { Doppler frequency shift } \\
\qquad(\mathrm{kHz})\end{array}$} \\
\hline & & $\begin{array}{c}1 \sigma \\
\text { error }\end{array}$ & $\begin{array}{c}\mathrm{JPL}^{a} \\
\text { catalog }\end{array}$ & Other $^{b}$ \\
\hline 2003-Feb.-23 & $\mathrm{HC}_{3} \mathrm{~N}(25 \rightarrow 24)$ & (12) & 48 & 42 \\
\hline 2004-Feb.-16 & & (8) & 14 & 8 \\
\hline 1999-Dec.-23 ${ }^{\dagger}$ & $\mathrm{HC}_{3} \mathrm{~N}(26 \rightarrow 25)$ & (4) & 47 & 42 \\
\hline 2003-Feb.-23 & $\mathrm{HC}_{3} \mathrm{~N}(12 \rightarrow 11)$ & (4) & 14 & 3 \\
\hline 2004-Feb.-16 & & (3) & 14 & 3 \\
\hline 1999-Dec.-23 ${ }^{\dagger}$ & $\mathrm{HC}_{3} \mathrm{~N}(10 \rightarrow 9)$ & (6) & -34 & -7 \\
\hline 2003-Mar.-10 & $\mathrm{CH}_{3} \mathrm{CN}(12 \rightarrow 11$ & (21) & -59 & 5 \\
\hline 2004-Feb.-17 & $K=3)$ & (28) & -50 & -4 \\
\hline 1999-Dec.-24 & & (7) & -43 & -11 \\
\hline 2003-Mar.-10 & $\mathrm{HCN}(1 \rightarrow 0)$ & (12) & 19 & - \\
\hline 2004-Feb.-17 & & (6) & 0 & - \\
\hline 1999-Dec.-24 & & (10) & -8 & - \\
\hline
\end{tabular}

Notes: Measured lineshift errors are in parentheses (third column).

Doppler shifts have been calculated from 2 sets of reference frequencies:

${ }^{a}$ The frequencies used in Col. 4 are from the JPL catalog (Pickett et al. 1998).

${ }^{b}$ The frequencies used in Col. 5 have been directly computed from the ground state rotational parameters of $\mathrm{HC}_{3} \mathrm{~N}$ and $\mathrm{CH}_{3} \mathrm{CN}$ given by Mbosei et al. (2000) and Pearson \& Muller (1996), respectively.

$\dagger$ Single-dish measurements obtained with the IRAM 30-m telescope in 1999 are listed for a quantitative comparison.

Table 4. Doppler velocity shift measurements.

\begin{tabular}{|c|c|c|c|}
\hline \multirow[t]{2}{*}{ Date } & \multirow[t]{2}{*}{ Transition } & \multicolumn{2}{|c|}{$\begin{array}{l}\text { Doppler velocity shift } \\
(\mathrm{m} / \mathrm{s})\end{array}$} \\
\hline & & East & West \\
\hline 2003-Feb.-23 & $\mathrm{HC}_{3} \mathrm{~N}(25 \rightarrow 24)$ & $-108(18)$ & $+3(17)$ \\
\hline 2004-Feb.-16 & & $-53(12)$ & $+5(13)$ \\
\hline 2003-Mar.-10 & $\mathrm{CH}_{3} \mathrm{CN}(12 \rightarrow 11)$ & $-65(36)$ & $+126(22)$ \\
\hline 2004-Feb.-17 & & $-1(47)$ & $+177(58)$ \\
\hline
\end{tabular}

Notes: Lines of $\mathrm{HC}_{3} \mathrm{~N}$ and $\mathrm{CH}_{3} \mathrm{CN}$ observed on Titan in 2003 and 2004 are used at spectral resolutions of 40 and $160 \mathrm{kHz}$, respectively (see text). Velocity uncertainties are in parentheses. The eastern and western equatorial positions correspond to RA, Dec offsets of $(+0.4,0.0)$ and $(-0.4,0.0)$ in the respective maps.

a 2.5-MHz-wide bandwidth (that was the same used to measure the Doppler lineshifts). These latter contribution functions best show the altitude ranges corresponding to our wind velocity estimates.

Finally, as can be seen in Fig. 9b, the mean probed altitudes are $450 \pm 100 \mathrm{~km}$ and $300 \pm 150 \mathrm{~km}$ for the $\mathrm{HC}_{3} \mathrm{~N}(J=25 \rightarrow$ $24)$ and $\mathrm{CH}_{3} \mathrm{CN}(J=12 \rightarrow 11)$ lines, respectively.

\section{Discussion - conclusion}

In 2003 and 2004 we mapped Titan at mm wavelengths, using interferometric techniques. Doppler lineshift measurements allowed unambiguous determination of the prograde wind flow during Titan's southern summer. Equatorial zonal wind velocities in the upper stratosphere and lower mesosphere are found to be $+160 \pm 60 \mathrm{~m} / \mathrm{s}$ and $+60 \pm 20 \mathrm{~m} / \mathrm{s}$ at altitudes of $300 \pm$ $150 \mathrm{~km}$ and $450 \pm 100 \mathrm{~km}$, respectively. We see a clear decrease in speed with increasing altitude. These observations confirm the superrotation of Titan's atmosphere, and the inferred mesospheric zonal velocity of $60 \mathrm{~m} / \mathrm{s}$ is the first determination for this altitude region. From our data, we cannot confirm that wind changes occurred between 2003 and 2004, even if leading and trailing hemispheres were observed. At 300-km altitude, our equatorial zonal wind velocity of $160 \mathrm{~m} / \mathrm{s}$ is more in agreement with high latitude jets (160-230 m/s) than with the equatorial zonal wind $(100-110 \mathrm{~m} / \mathrm{s})$ derived at $\sim 250 \mathrm{~km}$ from occultation measurements (Hubbard et al. 1993; Bouchez et al. 2003). Our value is very close to the equatorial zonal wind speed $(\sim 180 \mathrm{~m} / \mathrm{s})$ computed with a coupled dynamicsmicrophysics model proposed by Rannou et al. (2004).

Several efforts have been made to simulate Titan's atmospheric circulation. Hourdin et al. (1995) developed a GCM to study Titan's dynamics below $500 \mathrm{~km}$. Their model intrinsically produces a strong superrotation with prograde equatorial winds (i.e., a cyclostrophic circulation). The computed equatorial winds were almost in agreement with winds determined by Hubbard et al. (1993), although the high-latitude jets were not strong enough and latitudinal thermal contrasts were not well estimated. Hourdin et al. (1995) also indicated that to create and maintain a superrotation, upward meridional transport of the angular momentum was required and was dominated by large equator-to-pole Hadley cells. While Hourdin et al. (1995) predicted spontaneously superrotating winds in the stratosphere, the model of Tokano et al. (1999) reproduced superrotation only after applying artificial damping to the meridional stratospheric circulation. Furthermore, Müller-Wodarg et al. (2000) indicated that superrotating zonal winds in the stratosphere were set by the balance of pressure gradients, curvature and Coriolis forces. Recent simulations of Titan's GCM including haze effect (Rannou et al. 2002; Luz et al. 2003b; Rannou et al. 2004) predicted the haze accumulation at the pole well and provided the best simulations of the high-latitude northern jet, as well as the latitudinal thermal contrast. A seasonally varying haze appears to be the main source of the heating and cooling that drive atmospheric circulation. Dynamical transport can also be affected by photochemistry (Lebonnois et al. 2003).

In the thermosphere $(\sim 1000 \mathrm{~km})$, a scale analysis of the motion equation by Rishbeth et al. (2000) shows that day-night temperature differences of $20 \mathrm{~K}$ should induce thermospheric winds of $60 \mathrm{~m} / \mathrm{s}$. Moreover, Müller-Wodarg et al. (2000), who developed their GCM to study the thermosphere of Titan, argued that dynamics on this region, which is mainly driven by solar energy deposition, is characterized by the balance between pressure gradients and viscosity. Their calculations yielded thermospheric zonal winds of $\sim 60 \mathrm{~m} / \mathrm{s}$, consistent with 

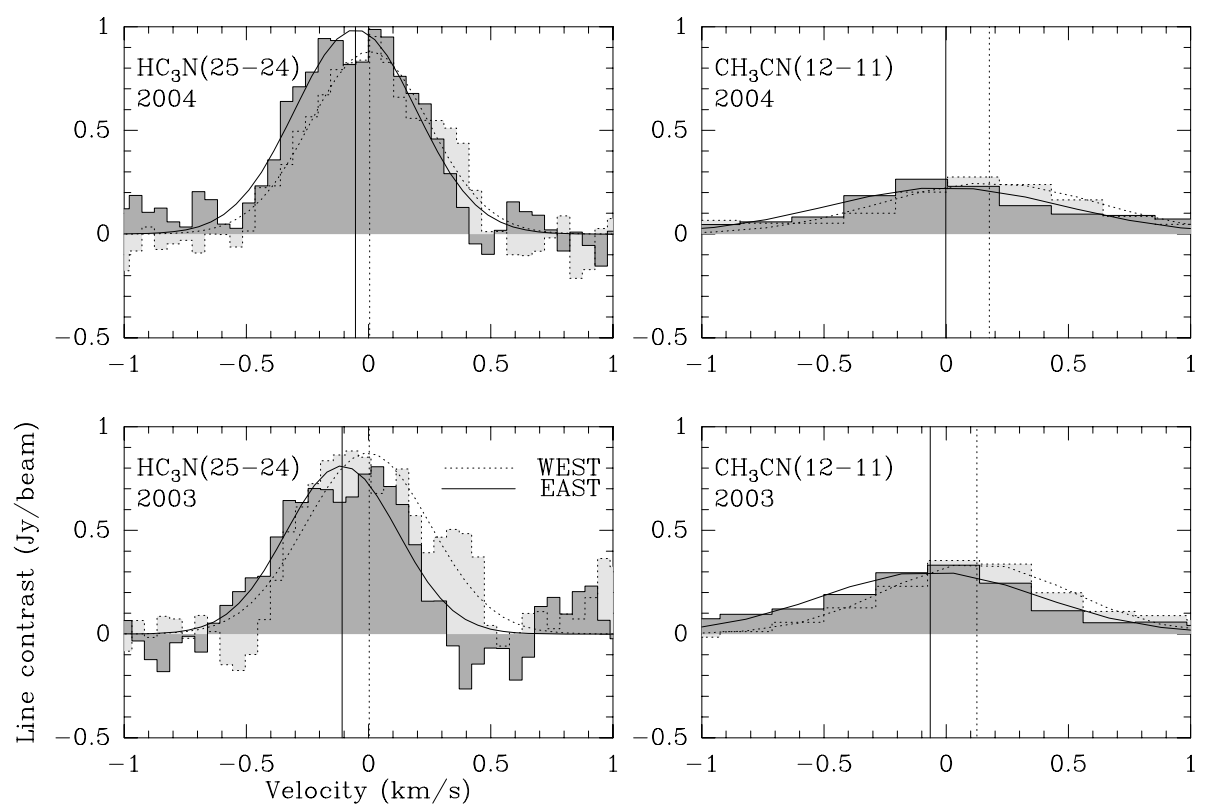

Fig. 6. $\mathrm{HC}_{3} \mathrm{~N}(25 \rightarrow 24)$ and $\mathrm{CH}_{3} \mathrm{CN}(12 \rightarrow 11)$ spectra observed at eastern and western equatorial limb positions corresponding to angular offsets $(+0.4,0.0)$ and $(-0.4,0.0)$. The spectral resolutions are 40 and $160 \mathrm{kHz}$ for $\mathrm{HC}_{3} \mathrm{~N}$ and $\mathrm{CH}_{3} \mathrm{CN}$, respectively. To enhance the line visibility, the $\mathrm{CH}_{3} \mathrm{CN}$ spectrum is made of the sum of the 4 strongest components (see text). The solid and dotted vertical bars show the respective centres of the Gaussian fits. The Doppler velocity shifts between the two equatorial positions are clearly visible. Precise velocity results are given in Table 4 .
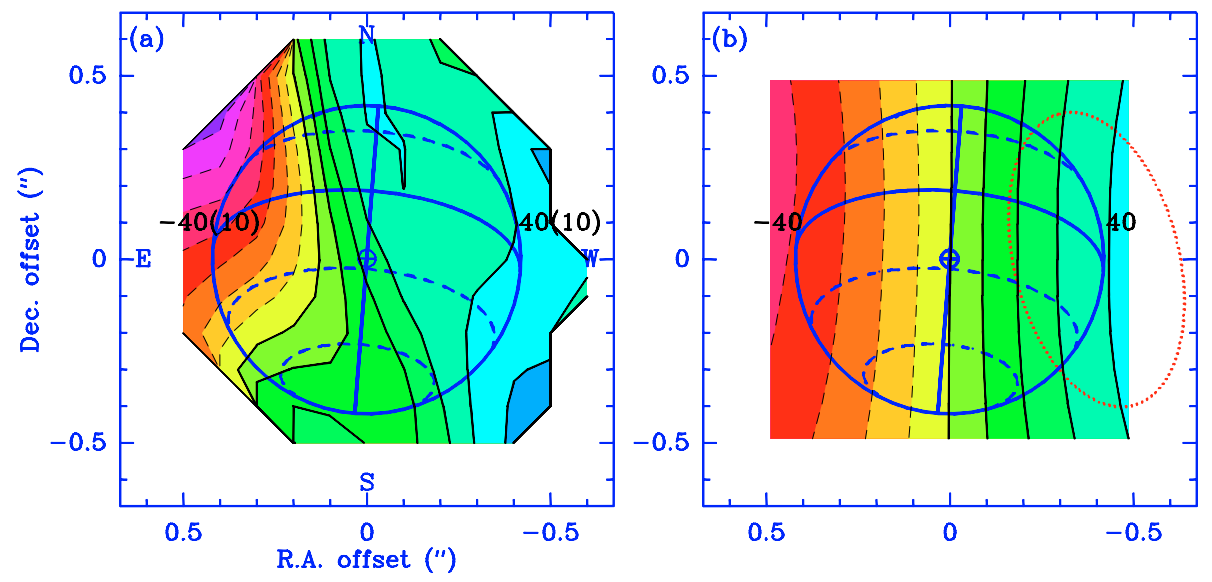

Fig. 7. a) (left panel) Doppler velocity map computed from the $\mathrm{HC}_{3} \mathrm{~N}(25 \rightarrow 24)$ data in Fig. 2. North is at the top. Titan's solid body diameter here is $0.84^{\prime \prime}$. Symbol $\oplus$ marks the sub-earth latitude. Positive velocities are indicated by solid black line, and negative velocities by dashed black lines. Contour levels are plotted in intervals of $10 \mathrm{~m} / \mathrm{s}$ and correspond to $1 \sigma$ noise. Doppler shifts values are indicated in $\mathrm{m} / \mathrm{s}$ for the equatorial region. b) (right panel) Simulated Doppler shift map for a rotating sphere with an equatorial prograde velocity of $+70 \mathrm{~m} / \mathrm{s}$. Convolution with a synthesized beam (shown by the dotted ellipse) has been introduced into the calculations. Contour levels are plotted from -40 to $40 \mathrm{~m} / \mathrm{s}$ by intervals of $10 \mathrm{~m} / \mathrm{s}$. A maximum Doppler shift of $40 \mathrm{~m} / \mathrm{s}$ computed at equatorial latitudes is in good agreement with the observed velocity map (left panel).

the results of Rishbeth et al. (2000). Using scale analysis and assuming a scale height $H \sim 100 \mathrm{~km}$, Müller-Wodarg \& Yelle (2002) derived a large vertical eddy coefficient $K$ on the order of $\sim 10^{9} \mathrm{~cm}^{2} \mathrm{~s}^{-1}$ in the thermosphere. Note that this value is consistent with the one deduced by Hidayat et al. (1997) from HCN observations. The vertical wind might therefore be responsible for the rapid vertical mixing in the upper atmosphere. Müller-Wodarg et al. (2000) found thermospheric solar driven wind speeds of 30 and $60 \mathrm{~m} / \mathrm{s}$ at solar minimum and maximum, respectively. The lower boundary in their models lies at $600 \mathrm{~km}$. If the winds, forced by circulation at the lower boundary, could indeed persist to the highest altitudes (Müller-Wodarg et al. 2000), then our results with mesospheric winds on the order of $60 \mathrm{~m} / \mathrm{s}$ might support such a coupling mechanism.

In the lower atmosphere down to the surface, Titan's dynamics is mostly influenced by Saturn's gravitational tide. Tidal oscillations can be caused by the $3 \%$ eccentricity of Titan's orbit around Saturn (Rishbeth et al. 2000). Tokano \& Neubauer (2002) investigated this possibility in detail and 

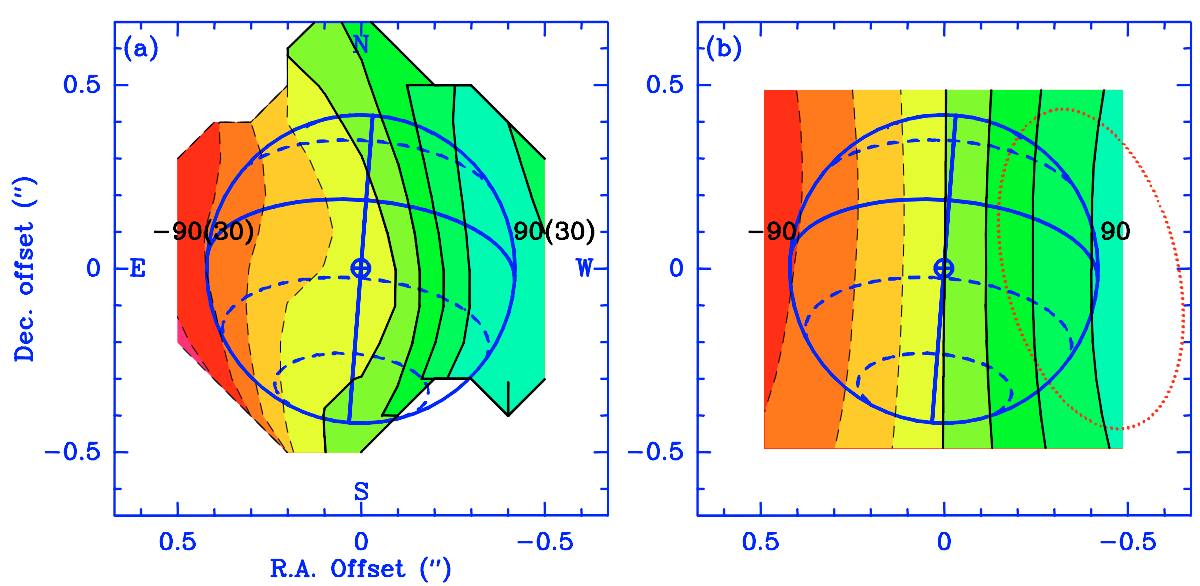

Fig. 8. Same as Fig. 7 for $\mathrm{CH}_{3} \mathrm{CN}$ data. An equatorial prograde velocity of $+170 \mathrm{~m} / \mathrm{s}$ is assumed in the simulated map b) shown in the right panel. The contour levels are plotted in intervals of $30 \mathrm{~m} / \mathrm{s}$. The maximum Doppler lineshift of $90 \mathrm{~m} / \mathrm{s}$ agrees well with the observed $\mathrm{CH}_{3} \mathrm{CN}_{\text {velocity }}$ map a).

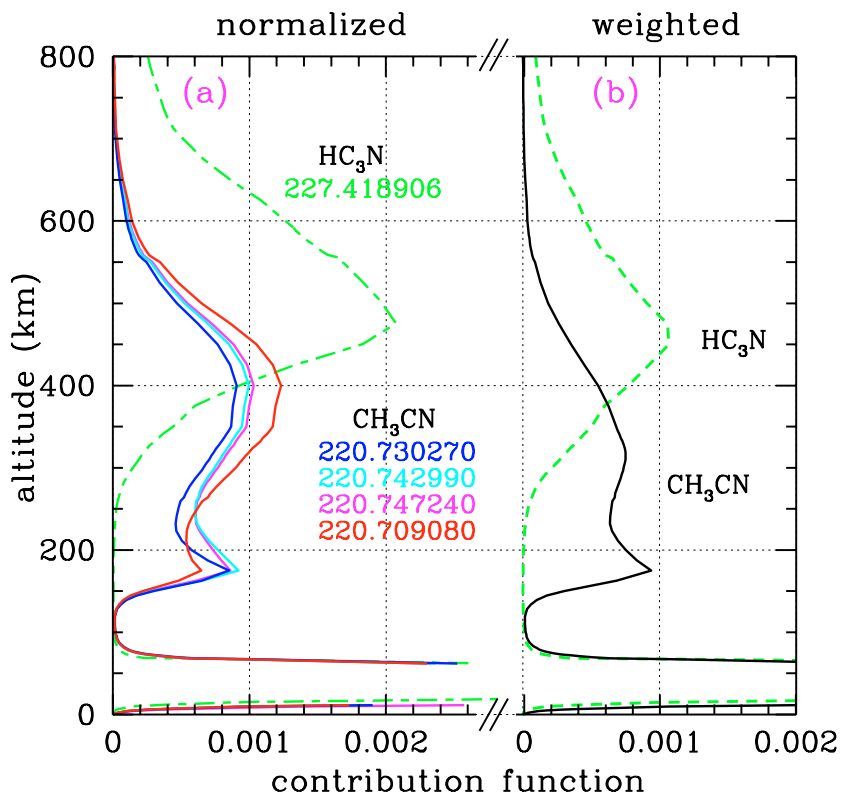

Fig. 9. a) Normalized contribution function computed for the transitions of $\mathrm{HC}_{3} \mathrm{~N}(J=25 \rightarrow 24)$ (dotted line) and $\mathrm{CH}_{3} \mathrm{CN}(J=$ $12 \rightarrow 11, K=0,1,2,3)$ (solid lines). Computations are done for the Titan's limb (angular offset of $0.4^{\prime \prime}$ from the centre), and with a circular beam of $0.6^{\prime \prime}$. Below 100-km altitude, the curves illustrate Titan's continuum formed in the troposphere, while in the upper atmosphere the contributions correspond to the lines observed in emission. b) Weighted contribution function (see text) computed for the transitions of $\mathrm{HC}_{3} \mathrm{~N}(J=25 \rightarrow 24)$ (dotted line) and the average of the 4 components $(K=0,1,2,3)$ of $\mathrm{CH}_{3} \mathrm{CN}(J=12 \rightarrow 11)$ (solid line).

found that near the surface the tidal winds indeed dominate the atmospheric flow and yield strong temporal and spatial variations. Zonal winds are found to be prograde since the tidal wind amplitude is smaller than the mean zonal wind.

The Huygens Doppler wind experiment (Bird et al. 2002) should yield very accurate wind velocity measurements in the low stratosphere and in the troposphere of Titan. Zonal winds will also be derived from thermal infrared maps observed during a long period of time by the CIRS spectrometer onboard the Cassini spacecraft (Kunde et al. 1996). Accompanying and follow-up ground-based observations will also be very useful. We can expect that in the next decade, wind structure will be determined by the ALMA array at a very high spatial resolution.

Acknowledgements. We wish to thank D. Downes for a careful reading of the manuscript and the PdBI operators for their special efforts during these planetary observations. Observations were carried out with the IRAM Plateau de Bure Interferometer. IRAM is supported by INSU/CNRS (France), MPG (Germany) and IGN (Spain).

\section{References}

Bevington, P. R., \& Robinson, D. K. 1992, Data Reduction and Error Analysis for the Physical Sciences, 2nd Ed. (McGraw-Hill)

Bird, M. K., Dutta-Roy, R., Heyl, M., et al. 2002, Space Sci. Rev., 104, 613

Bouchez, A. H., Brown, M. E., Troy, et al. 2003, BAAS, 35, 928

Brown, F. X., Dangoisse, D., \& Demaison, J. 1988, J. Mol. Spec., 129, 483

Flasar, F. M., Samuelson, R. E., \& Conrath, B. J. 1981, Nature, 292, 693

Flasar, F. M. 1998, Planet. Space Sci., 46, 1125

Guilloteau, S., Delannoy, J., Downes, D., et al. 1992, A\&A, 262, 624

Hidayat, T., Marten, A., Bézard, et al. 1997, Icarus, 126, 170

Hidayat, T., Marten, A., Bézard, et al. 1998, Icarus, 133, 109

Hourdin, F., Talagrand, O., Sadourny, R., et al. 1995, Icarus, 117, 358

Hubbard, W. B., Sicardy, B., Miles, R., et al. 1993, A\&A, 269, 541

Kostiuk, T., Fast, K. E., Livengood, et al. 2001, Geophys. Res. Lett., 28,2361

Kunde, V., Ade, P., Barney, R., et al. 1996, Cassini infrared Fourier spectroscopic investigation. In Cassini/Huygens: A Mission to the Saturnian Systems, Proc. SPIE, 2803, 162

Lebonnois, S., Hourdin, F., Rannou, P., et al. 2003, Icarus, 163, 164 
Luz, D., Courtin, R., Gautier, D., et al. 2003a, BAAS, 35, 927

Luz, D., Hourdin, F., Rannou, P., \& Lebonnois, S. 2003b, Icarus, 166, 343

Marten, A., Hidayat, T., Biraud, Y., \& Moreno, R. 2002, Icarus, 158, 532

Marten, A., \& Moreno, R. 2003, BAAS, 35, 952

Mbosei, L., Fayt, A., Dréan, P., \& Cosléou, J. 2000, J. Mol. Structure, 517-518, 271

Moreno, R., Marten, A., Biraud, Y., et al. 2001, Planet. Space Sci., 49, 473

Muller, H. S. P., Thorwirth, S., Roth, D. A., \& Winnewisser, G. 2001, A\&A, 370, L49

Müller-Wodarg, I. C. F., Yelle, R. V., Mendillo, M., Young, L. A., \& Aylward, A. D. 2000, J. Geophys. Res., 105(A9), 20833

Müller-Wodarg, I. C. F., \& Yelle, R. V. 2002, Geophys. Res. Lett., 29, 54
Nguyen-Van-Thanh, \& Rossi, I. 1993, J. Mol. Spec., 157, 68

Pearson, J. C., \& Muller, H. S. P. 1996, ApJ, 471, 1067

Pickett, H. M., Poynter, R. L., Cohen, E. A., et al. 1998, J. Quant. Spec. Radiat. Transf., 60, 883

Rannou, P., Hourdin, F., \& McKay, C. P. 2002, Nature, 418, 853

Rannou, P., Hourdin, F., McKay, C. P., \& Luz, D. 2004, Icarus, 170, 443

Rishbeth, H., Yelle, R. V., \& Mendillo, M. 2000, Planet. Space Sci., 48,51

Schubert, G. 1983, General circulation and the dynamical state of the Venus atmosphere. Venus (University of Arizona Press), 681

Tokano, T., Neubauer, F. M., Laube, M., \& McKay, C. P. 1999, Planet. Space Sci., 47, 493

Tokano, T., \& Neubauer, F. M. 2002, Icarus, 158, 499 